\title{
EFECTO ANTIOXIDANTE DE FRUTAS Y HORTALIZAS DE LA ZONA CENTRAL DE CHILE
}

\section{ANTIOXIDANT EFFECT OF FRUITS AND VEGETABLES OF CENTRAL REGION OF CHILE}

Iván Palomo G. (1), Margarita Gutiérrez C. (2), Luís Astudillo S. (2), Carolina Rivera S. (2) Constanza Torres U.(1), Luís Guzmán J. (1), Rodrigo Moore-Carrasco (1), Gilda Carrasco S. (3) Marcelo Alarcón L. (1)

(1) Programa de Investigación en Factores de Riesgo de Enfermedades Cardiovasculares (PIFRECV), Departamento de Bioquímica Clínica e Inmunohematología, Facultad de Ciencias de la Salud,

Universidad de Talca. Talca, Chile.

(2) Laboratorio de Síntesis Orgánica, Instituto de Química de Recursos Naturales,

Universidad de Talca. Talca, Chile.

(3) Departamento de Horticultura, Facultad de Ciencias Agrarias,

Universidad de Talca. Talca, Chile.

\begin{abstract}
The reactive oxygen species (ERO) cause cell damage that can be expressed as a disease, including cardiovascular disease (CVD) and other non transmissible chronic diseases. The human body has antioxidant systems, some come from the diet, especially fruits and vegetables, and others are generated by the same organism as an endogenous way. The aim of this study was to determine the in vitro antioxidant capacity of some fruits and vegetables that are consumed in the Maule Region, Chile. Eleven species of fruits and 16 of vegetables used in the study were obtained at the Regional Center of Fruits and Vegetables of Talca during the harvest season, and different aqueous and methanol extract were obtained. The antioxidant activity was determined by purple discoloration of the radical 2,2-diphenyl-1-picryl hidrazilo hydrated (DPPH). In general the antioxidant activity was slightly higher in fruits $(70,3 \% ; 87,7-54,3 \%)$ than in vegetables $(54,7 \% ; 44,3-79,7 \%)(p \leq 0.003)$ and it was dependent on the concentration of the extract. In the tests at $1000 \mu \mathrm{g} / \mathrm{m}$, most fruits showed a higher percentage of discoloration over to 60\%; those that showed higher antioxidant activity were raspberry, strawberry and kiwi. Among vegetables, those showing higher antioxidant activity were tomato, followed by melon pear, red beet, melon (type tuna), sweet pepper and watermelon. The antioxidant activity observed in most fruits and vegetables could be assessed as a scientific argument to make known in promotional campaigns in national consumption and the horticultural products exports.
\end{abstract}

Key words: fruits; vegetables; antioxidants; DPPH.

Este trabajo fue recibido el 2 de Diciembre de 2008 y aceptado para ser publicado el 1 de Abril de 2009.

\section{INTRODUCCIÓN}

Las especies reactivas del oxígeno (ERO) son agentes oxidantes que tienen o son fácilmente convertidos en radicales libres, los que son átomos o grupos de átomos inestables de alta energía que poseen uno o más electrones desapareados o libres en sus orbitales exteriores, por lo que son muy reactivos ya que tienden a captar un electrón de moléculas estables con el fin de alcanzar su estabilidad electroquímica (1). Las ERO, cuyo origen es endógeno y exógeno (contaminación atmosférica, humo de cigarrillos, aceites vegetales hidrogenados y ácidos grasos trans), cuando no son suficientemente neutralizados oxidan macromoléculas fundamentales en las células, como fosfolípidos, proteínas y $\mathrm{ADN}$, lo que causa alteraciones celulares que se pueden expresar en patologías, entre ellas enfermedades cardiovasculares (ECV) y otras enfermedades crónicas no transmisibles $(2,3)$. El organismo humano cuenta con sistemas antioxidantes 
endógenos y exógenos; estos últimos provienen de la dieta, especialmente de frutas y hortalizas; algunos son de tipo nutriente (vitaminas $\mathrm{E}$ y C) y otros no nutrientes (compuestos fenólicos y polifenoles)(4). En la naturaleza solo los vegetales sintetizan antioxidantes, pero no todos los producen en la misma cantidad y tipo. El propósito de este estudio fue conocer la capacidad antioxidante de algunas frutas y hortalizas pertenecientes a diversas familias botánicas que se producen y consumen en la Región del Maule.

\section{MATERIALES Y MÉTODOS Material vegetal}

Se obtuvieron especies hortofrutícolas de diferentes familias botánicas con el propósito de obtener extractos utilizando sus órganos comestibles en estado fresco. Estas especies fueron adquiridas en el Centro Regional de Abastecimiento de Talca, Región del Maule, en sus respectivas épocas de cosecha. Las frutas estudiadas, clasificadas de acuerdo a sus familias botánicas fueron: Familia Rosácea: ciruelas, frambuesas, frutillas, manzanas (Galaxy, Golden, Granny Smith, Royal Gala y Scarlet), peras (Abate, Asiática y de agua); Familia Auranciácea: pomelos y Familia Actinidiácea; uvas (tipo Curtiduría, País, Red Globe, Sultanina, Torontel) y kiwis.

Las familias botánicas de hortalizas evaluadas fueron familia Quenopodiácea: acelga, betarraga y espinaca; familia crucífera: brócoli, coliflor y repollo; familia Solanácea: tomate (tipo Racimo y tipo Talquino), berenjena, pepino dulce, pimentón rojo (tipo Talquino y tipo Zafiro) (familia Solanácea); familia Aliáceas: ajo, cebolla (tipo de guarda y temprana) y cebollín; familia Compuesta: lechuga (tipos Costina, Española e Iceberg; melón (tipos Plátano y Tuna), sandía, familia Cucurbitácea: pepino de ensalada; familia Fabácea; porotos tipo Granados y tipo Verdes y familia Umbelífera: zanahoria.

\section{Preparación de extractos}

A partir de 2-3 frutas y hortalizas, por especie y según el caso variedad, se prepararon extractos acuosos y metanólicos, dependiendo de las características fisiológicas de la muestra a tratar, a fin de evaluar rendimiento y bioactividad en cada caso.

Extracción acuosa.Las frutas y hortalizas seleccionadas fueron lavadas, peladas y cortadas en trozos pequeños, luego mediante prensa manual se procedió a extraer el jugo (30-55 ml), el cual fue doblemente filtrado sobre papel filtro y posteriormente congelado a $-20^{\circ} \mathrm{C}$ para ser liofilizado (Freezone 6, Labconco, USA). Los liofilizados obtenidos fueron pesados en balanza analítica y luego sellados y almacenados a $-70^{\circ} \mathrm{C}$ hasta su uso.
Extracción metanólica. La muestra (150 g) fue lavada, pelada y cortada, en trozos muy pequeños, se añadió $150 \mathrm{ml}$ de metanol (Analytic, Importado por Arquimed, Santiago, Chile), esta mezcla fue sonicada (Transsonic 700/H, Elma-Hans Schmidbauer, Alemania), por 5 minutos y luego filtrada. Este procedimiento se repitió a fin de obtener una mayor extracción. Los filtrados obtenidos fueron llevados a sequedad a presión reducida $\left(40^{\circ} \mathrm{C}, 90-180 \mathrm{rpm}\right.$, Laborota 4001 , Heidolph, Alemania o RE 111-B461, BÜCHI Labortechnik AG, Holanda). Los extractos fueron almacenados en oscuridad a $-20^{\circ} \mathrm{C}$ para posteriormente liofilizar, sellar y almacenar a $-70^{\circ} \mathrm{C}$ hasta su empleo.

\section{Evaluación de la actividad antioxidante}

La actividad antioxidante de los extractos se determinó usando la prueba de decoloración del radical violeta 2,2-difenil-1-picril hidrazilo hidratado (DPPH, $\left.\mathrm{C}_{18} \mathrm{H}_{12} \mathrm{~N}_{5} \mathrm{O}_{6}\right)(5,6)$. La actividad antioxidante se mide en términos de donar hidrógeno o de la capacidad atrapadora de radicales. La reducción y estabilización del DPPH por los antioxidantes da lugar a la decoloración de éste, produciendo una disminución de la absorbancia a $517 \mathrm{~nm}$ y se expresa como porcentaje de decoloración de la solución radicalaria a una concentración dada. Los extractos de cada fruta y hortaliza se disolvieron en un mínimo de DMSO y se llevo a una concentración de análisis de $10 \mathrm{mg} / \mathrm{mL}$ en agua (solución stock). A partir de ésta se realizaron diluciones que permitiesen obtener una concentración final en el ensayo de 1000, 500, 100 y $50 \mu \mathrm{g} / \mathrm{mL}$. Estas soluciones $(0,35 \mathrm{~mL})$ se mezclaron con $0,7 \mathrm{~mL}$ de solución metanólica de DPPH (20 $\mu \mathrm{g} /$ $\mathrm{mL}$ ) y se dejó reposar durante 5 minutos, para producir reacción. Cada extracto fue evaluado en triplicado; la absorbancia se registró en un espectrofotómetro (Thermo Electron Corporation, Multiskan, Finland) a 517nm, descontado la lectura de la muestra pura y comparando su valor con un blanco ausente de muestra. El porcentaje de decoloración se estableció según la siguiente fórmula: 1-(DO muestra)/DO blanco x 100. El grado de decoloración indica la eficacia de los extractos estudiados como atrapadores de radical libre, por lo tanto, un porcentaje de decoloración de 100 indica la máxima capacidad atrapadora y un valor cercano a 0 indica una nula capacidad. Como compuesto de referencia se utilizó quercetina y catequiza en un rango de concentración entre 1,25 a 7,5 $\mu \mathrm{g} / \mathrm{mL}$. Los valores son presentados como promedio \pm desviación estándar de tres determinaciones.

Análisis estadístico. Los resultados de la actividad antioxidante fueron expresados como mediana y rango mínimo y máximo de 3 determinaciones y en este caso las comparaciones estadísticas se realizaron con la 
prueba no paramétrica Kruskall-Wallis. Las comparaciones entre grupos de frutas se realizaron con T-test. En todos los casos se utilizó el programa computacional SPSS 15.0 (Statistical Product and Service Solutions). Valores $\mathrm{p}<0.05$ fueron considerados estadísticamente significativos.

\section{RESULTADOS}

El rendimiento de los extractos acuosos y metanólicos de las frutas fue de $14,5 \pm 4,5 \%$ y $11,2 \pm 4,4 \%$, respectivamente; en el caso de las hortalizas fue $7,2 \pm 2,8 \%$ y $4,7 \pm 2,4 \%$, respectivamente.

La actividad antioxidante, expresada como porcentaje de decoloración de la solución radicalaria de DPPH, por parte de extractos a $1000,500,100$ y $50 \mu \mathrm{g} / \mathrm{mL}$ de concentración final, se muestra en la tabla 1 para frutas y en la tabla 2 para hortalizas.

En general la actividad antioxidante, en extractos metanólicos, fue mayor en frutas $(70,3 \% ; 87,7-54,3 \%$ de decoloración) que en hortalizas $(54,7 \% ; 44,3-79,7 \%)$ (p $0.003)$. Por otra parte, la actividad antioxidante, tanto en frutas como en hortalizas y en ambos tipos de extractos, fue dependiente de la concentración empleada. En dicho contexto, el análisis que se hace a continuación se refiere a los resultados obtenidos con la mayor concentración de extracto utilizada, $1000 \mu \mathrm{g} / \mathrm{mL}$.

En frutas, en las que en general se obtuvo extractos acuoso y metanólico, la actividad antioxidante en las

\section{TABLA 1}

\section{Porcentaje de decoloración de solución de DPPH en extractos acuosos (jugo) y metanólicos (pulpa) de frutas.}

\begin{tabular}{|c|c|c|c|c|c|c|}
\hline & \multicolumn{2}{|c|}{$1000 \mu \mathrm{g} / \mathrm{ml}$} & \multicolumn{2}{|c|}{$500 \mu \mathrm{g} / \mathrm{ml}$} & \multicolumn{2}{|c|}{$100 \mu \mathrm{g} / \mathrm{ml}$} \\
\hline Ciruela & $67(65-70)$ & $71(70-73)$ & $34(32-40)$ & $47(45-50)$ & $8(8-15)$ & $6(5-12)$ \\
\hline Frambuesa & $82(75-85)$ & $78(75-80)$ & $55(54-64)$ & $42(35-42)$ & $22(20-25)$ & $15(13-15)$ \\
\hline Frutilla & $84(80-85)$ & $88(85-90)$ & $50(50-55)$ & $50(50-55)$ & $15(14-15)$ & $28(15-30)$ \\
\hline Kiwi & $80(80-88)$ & $78(75-80)$ & $48(45-50)$ & $50(50-55)$ & $15(10-19)$ & $12(10-15)$ \\
\hline \multicolumn{7}{|l|}{ Manzana } \\
\hline Galaxy & $68(65-70)$ & $58(57-65)$ & $48(43-50)$ & $38(38-45)$ & $14(12-18)$ & $7(5-10)$ \\
\hline Golden & $70(68-80)$ & $73(72-80)$ & $42(40-45)$ & $37(35-42)$ & $26(20-30)$ & $9(8-10)$ \\
\hline Granny Smith & $58(58-65)$ & $65(65-72)$ & $35(32-37)$ & $35(32-37)$ & $20(15-22)$ & $20(15-22)$ \\
\hline Royal Gala & $68(60-68)$ & $68(68-75)$ & $45(43-48)$ & $47(40-48)$ & $12(11-12)$ & $8(6-10)$ \\
\hline Scarlet & $60(55-63)$ & $63(55-63)$ & $38(35-40)$ & $35(32-37)$ & $9(8-10)$ & $11(8-12)$ \\
\hline \multicolumn{7}{|l|}{ Pera } \\
\hline Abaté & - & $55(53-55)$ & - & $24(23-28)$ & - & $14(13-18)$ \\
\hline Asiática & $47(46-50)$ & $60(58-63)$ & $22(20-25)$ & $36(35-40)$ & $8(7-8)$ & $8(7-8)$ \\
\hline De Agua & $54(52-58)$ & $60(64-61)$ & $28(28-35)$ & $32(30-36)$ & $14(12-15)$ & $5(4-5)$ \\
\hline Pomelo & $66(63-67)$ & $78(70-80)$ & $48(40-48)$ & $47(45-50)$ & $15(13-16)$ & $8(7-8)$ \\
\hline \multicolumn{7}{|l|}{ Uva } \\
\hline País & - & $67(66-70)$ & - & $47(45-50)$ & - & $11(10-15)$ \\
\hline Red Globe & $64(64-68)$ & $60(47-62)$ & $39(38-44)$ & $45(40-50)$ & $17(15-22)$ & $12(10-15)$ \\
\hline Curtiduría & $75(65-75)$ & $73(65-75)$ & $48(47-50)$ & $45(39-50)$ & $15(10-15)$ & $18(18-23)$ \\
\hline Sultanina & $72(65-73)$ & - & $55(49-60)$ & - & $14(12-18)$ & - \\
\hline Torontel & $65(63-68)$ & - & $44(44-45)$ & - & $8(7-12)$ & - \\
\hline
\end{tabular}


mismas especies y variedades fueron similares (p NS). En frutas, la mayoría mostró un porcentaje de decoloración (actividad antioxidante) superior al $60 \%$ y en el caso de frambuesa, frutilla y kiwi en su conjunto (mediana $77,7 \%$; rango $77,7-87,7 \%$ ) fue significativamente mayor que el resto de las frutas $(67,5 ; 54,3-77,7)(\mathrm{p}<0.01)$.

Entre las hortalizas, los extractos metanólicos de tomates, pepino dulce, betarraga, melón tuna, pimentón y sandía presentaron actividad antioxidante significativamente mayor $(76,5 \% ; 73,3-79,7 \%)$ que el resto de las

\section{TABLA 2}

Porcentaje de decoloración de solución de DPPH para extractos metanólicos de hortalizas.

\begin{tabular}{|c|c|c|c|}
\hline & $1000 \mu \mathrm{g} / \mathrm{ml}$ & $500 \mu \mathrm{g} / \mathrm{ml}$ & $100 \mu \mathrm{g} / \mathrm{ml}$ \\
\hline Acelga & $58(48-60)$ & $30(24-30)$ & $10(5-10)$ \\
\hline Ajo & $50(45-50)$ & $26(25-32)$ & $10(8-10)$ \\
\hline Berenjena & $45(39-50)$ & $30(25-30)$ & $5(5-7)$ \\
\hline Betarraga & $76(70-80)$ & $55(48-55)$ & $20(20-25)$ \\
\hline Brócoli & $50(45-50)$ & $42(40-42)$ & $11(9-12)$ \\
\hline \multicolumn{4}{|l|}{ Cebolla } \\
\hline Guarda & $44(43-48)$ & $28(26-35)$ & $7(6-10)$ \\
\hline Verano & $50(45-50)$ & $28(18-29)$ & $5(5-7)$ \\
\hline Cebollín & $59(50-60)$ & $28(25-30)$ & $8(6-10)$ \\
\hline Coliflor & $45(43-45)$ & $28(18-29)$ & $12(7-15)$ \\
\hline Espinaca & $52(50-55)$ & $38(30-38)$ & $16(12-18)$ \\
\hline \multicolumn{4}{|l|}{ Lechuga } \\
\hline Costina & $45(43-48)$ & $28(25-30)$ & $10(8-10)$ \\
\hline Española & $54(50-60)$ & $25(20-25)$ & $7(3-10)$ \\
\hline Iceberg & $55(53-55)$ & $38(30-38)$ & $12(12-13)$ \\
\hline Melón Plátano & $57(55-60)$ & $35(32-38)$ & $12(11-12)$ \\
\hline Tuna & $75(74-80)$ & $48(45-50)$ & $10(5-10)$ \\
\hline \multicolumn{4}{|l|}{ Pepino } \\
\hline Dulce & $80(72-81)$ & $38(30-38)$ & $8(7-8)$ \\
\hline Ensalada & $55(51-58)$ & $33(30-35)$ & $5(5-6)$ \\
\hline \multicolumn{4}{|l|}{ Pimentón } \\
\hline Talquino & $70(70-80)$ & $35(35-37)$ & $20(13-22)$ \\
\hline Zafiro & $80(70-80)$ & $45(36-45)$ & $14(12-20)$ \\
\hline \multicolumn{4}{|l|}{ Porotos } \\
\hline Granados & $52(50-55)$ & $30(30-35)$ & $13(10-15)$ \\
\hline Verdes & $54(50-60)$ & $30(25-30)$ & $8(5-10)$ \\
\hline Repollo & $55(53-58)$ & $34(32-38)$ & $14(10-15)$ \\
\hline Sandía & $74(74-75)$ & $41(40-45)$ & $15(10-15)$ \\
\hline \multicolumn{4}{|l|}{ Tomate } \\
\hline Racimo & $78(76-80)$ & $50(50-55)$ & $19(18-24)$ \\
\hline Talquino & $82(72-85)$ & $55(50-55)$ & $15(13-18)$ \\
\hline
\end{tabular}


hortalizas estudiadas $(52,3 \% ; 44,3-56,3 \%)(\mathrm{p}<0.001)$. Las berenjenas, cebollas y coliflor fueron las hortalizas que mostraron el menor porcentaje de decoloración $(\sim 45 \%)$. En el caso de los extractos de melón, tomates, sandía y pepino dulce, también se evaluaron sus extractos acuosos, los que si bien presentaron actividad antioxidante levemente inferior en comparación con extractos metanólicos de los mismos, la diferencia no fue estadísticamente significativa.

Los compuestos catequina y quercetina, utilizados como referencia, presentaron una decoloración de $50 \%$ a la concentración final en el ensayo de 1,94 $\pm 3,2$ y 1,0 00,9 $\mu \mathrm{g} / \mathrm{mL}$, respectivamente.

\section{DISCUSIÓN}

Es reconocido que las frutas (7) y las hortalizas $(8,9)$ presentan compuestos que actúan como antioxidantes y que su consumo diario disminuye el riesgo de desarrollar ECV (10) y otras enfermedades crónicas no transmisibles (11). En ese contexto resulta importante conocer las propiedades antioxidantes de frutas y hortalizas que se producen y consumen en la Región del Maule.

Rendimiento de la extracción. Al comparar los métodos de extracción y analizar el porcentaje de rendimiento, observamos una variación poco significativa entre ambos, por lo cual el tipo de método no afectaría la cantidad de extraíbles para cada muestra. La extracción metanólica sería mas ventajosa en comparación a la acuosa debido a la dificultad que presenta la eliminación de agua de la muestra así preparada, teniendo cuidado con la temperatura de trabajo y exposición a la luz de la muestra, ya que éstas podrían degradarse por efecto de dichas variables.

Actividad antioxidante. Todas las frutas y hortalizas estudiadas presentaron actividad antioxidante, destacando frambuesa, frutilla, kiwi, tomates, pepino fruta, betarraga, melón tuna, pimentón y sandia. En la mayoría de los casos los extractos de pulpa, respecto a los extractos acuosos, presentaron una actividad levemente mayor.

A nivel mundial los estudios realizados han mostrado diversidad en las propiedades antioxidantes de las frutas y vegetales. Así Wu et al (12), encontraron que las paltas y las frambuesas presentan mayor capacidad antioxidante que los brócolis y zanahorias, resultados han sido confirmados por otros autores $(13,14)$. En Chile se han realizado estudios tendientes a caracterizar la capacidad antioxidante de frutas y hortalizas. Así Araya et al (15) analizaron diferentes frutas y verduras cultivadas en el país, encontrando que el maqui, berries (frutilla y zarzamora), zanahoria y ají rojo presentaban la mayor capacidad antioxidante. Dicha información ha sido confirmada por Speisky et al (16).

Las diferencias en los resultados encontrados por los diferentes investigadores podrían explicarse en parte por las diferentes variedades botánicas de las frutas y las verduras utilizadas, como también diferencias del lugar y las condiciones de cultivo, de almacenamiento, y de procesamiento, además de las diferentes metodologías utilizadas en la medición de la capacidad antioxidante (17). A nivel nacional el estudio de la actividad antioxidante del tomate no ha sido relevante; la alta actividad antioxidante encontrada por nosotros ha sido descrita en estudios realizados en Taiwain (18) y España $(19,20)$.

En estudios futuros seria de interés obtener mayor cantidad de masa de aquellos extractos que presentaron mejor actividad antioxidante, a fin de realizar aislamiento bioguiado de las moléculas que presentan dicha actividad, y así identificar químicamente el principio activo.

Chile, y en particular la zona central, tienen una economía mayoritariamente agraria, por lo tanto el hecho de que frutas y hortalizas, además de ser la principal fuente de vitaminas, minerales, fitoquímicos y fibra, presenten actividad antioxidante $(21,22)$ y antitrombótico (23) es destacable, ya que este efecto positivo en la salud humana, entrega un valor agregado a su producción, y por ende a su consumo interno y exportación. Se estima que el consumo de de frutas y verduras a nivel nacional es de $\sim 200 \mathrm{~g} /$ persona/día, no obstante lo recomendado es de $\sim 400 \mathrm{~g} /$ persona/día (24). En este sentido, es importante promover el desarrollo de nuevos mercados internos y externos e incrementar el consumo local mediante promoción y campañas destinadas a fomentar la alimentación sana y relacionar ésta con la ingesta de frutas y hortalizas, así como la campaña "5 al día" iniciada en el año 2007 por el Ministerio de Salud y Agricultura de Chile y el Instituto de Nutrición y Tecnología de los Alimentos, destinada a promover el consumo de a lo menos cinco frutas y verduras diarias en la población $(25,26)$.

\section{RESUMEN}

Las especies reactivas del oxígeno (ERO) causan daño celular que se puede expresar como patología, tales como las enfermedades cardiovasculares (ECV) y otras enfermedades crónicas no transmisibles. El organismo humano cuenta con sistemas antioxidantes; algunos provienen de la dieta, especialmente de frutas y hortalizas, otros los genera el mismo organismo de manera endógena. El objetivo de este estudio fue conocer la capacidad antioxidante in vitro de algunas frutas y hortalizas que se consumen en la Región del Maule de Chile. Las once especies de frutas y 16 de hortalizas empleadas en el estudio fueron adquiridas en el Centro Regional de 
Abastecimiento de Talca, en época de cosecha; a partir de ellas se obtuvo extractos acuosos y metanólicos. La actividad antioxidante se determinó mediante decoloración del radical violeta 2,2-difenil-1-picril hidrazilo hidratado (DPPH). En general la actividad antioxidante, en extractos metanólicos, fue mayor en frutas $(70,3 \%$; $87,7-54,3 \%$ de decoloración) que en hortalizas $(54,7 \%$; $44,3-79,7 \%)(\mathrm{p}<0.003)$ y dependiente de la concentración del extracto evaluado. En los ensayos a 1000 $\mu \mathrm{g} / \mathrm{m}$, la mayoría de las frutas mostraron un porcentaje de decoloración superior al $60 \%$ y las que presentaron mayor actividad antioxidante fueron frambuesa, frutilla y kiwi. Entre las hortalizas, las que presentaron mayor actividad antioxidante, fueron los tomates seguidos por pepino dulce, betarraga, melón tuna, pimentón y sandía. La actividad antioxidante observada en la mayoría de las frutas y hortalizas evaluadas podría ser un argumento científico a dar a conocer en las campañas de promoción del consumo interno y en la exportación de productos hortofrutícolas.

Palabras claves: frutas; hortalizas; antioxidantes; DPPH.

Dirigir la correspondencia:

Profesor

Iván Palomo González, PhD.

Departamento de Bioquímica Clínica

e Inmunohematología,

Facultad Ciencias de la Salud,

Universidad de Talca, Talca, Chile.

Casilla: 747

Talca, Chile.

E-mail: ipalomo@utalca.cl

Fono: 56-71-200493

Fax : 56-71-200488

Agradecimientos: Al Programa de Investigación en Factores de Riesgo de Enfermedades Cardiovasculares (PIFRECV), a los proyectos Anillo ACT-38 y PBCT PSD-16, y la Dirección de Investigación de la Universidad de Talca.

\section{BIBLIOGRAFÍA}

1. Avello M SM. Radicales libres, antioxidantes naturales y mecanismos de protección. Atenea. 2006: pp 161-172.

2. Meydani M. Antioxidants and cognitive function. Nutr Rev 2001;59:S75-80; discussion S80-72.

3. Zamora S J. Antioxidantes: micronutrientes en lucha por la salud. Rev Chil Nutr 2007;34:17-26.

4. La Vecchia C, Altieri A, Tavani A. Vegetables, fruit, antioxidants and cancer: a review of Italian studies.
Eur J Nutr 2001;40:261-267.

5. Viturro C, Molina A, Schmeda-Hirschmann G. Free radical scavengers from Mutisia friesiana (Asteraceae) and Sanicula graveolens (Apiaceae). Phytother Res 1999;13:422-424.

6. Galati EM, Mondello MR, Giuffrida D, Dugo G, Miceli N, Pergolizzi S, Taviano MF. Chemical characterization and biological effects of Sicilian Opuntia ficus indica (L.) mill. Fruit juice: antioxidant and antiulcerogenic activity. J Agric Food Chem. 2003;51:4903-4908.

7. Speisky H, Peña A, Gómez M, Fredes C, Hurtado M, Gotteland M, Brunser O. Antioxidants in Chilean berries. Acta Hort. 2008;777:485-492.

8. 8. Palma JM, Corpas FJ. Antioxidantes vegetales y su influencia en la dieta. . Horticultura. 2007;203:70-75.

9. Visioli F, Bogan S, Grande CG. Mediterranean food and health: building human evidence. J Phisiol Pharmacol 2005;56:37-49.

10. Adebawo O, Salau B, Ezima E, Oyefuga O, Ajani E, Idowu G, Famodu A, Osilesi O. Fruits and vegetables moderate lipid cardiovascular risk factor in hypertensive patients. Lipids Health Dis 2006;5:14.

11. Viviana V. Frutas y verduras en la prevención de enfermedades no contagiosas. Diabetes Voice. 2006;51:17-19.

12. Wu X, Gu L, Holden J, Haytowitz D, Gebhardt S, Beecher G, Prior R. Development of a database for total antioxidant capacity in foods: a preliminary study. J Food Composition Analysis. 2004;17:407422.

13. Yamamoto J, Naemura A, Ijiri Y, Ogawa K, Suzuki T, Shimada Y, Giddings JC. The antithrombotic effects of carrot filtrates in rats and mice. Blood Coagul Fibrinolysis. 2008;19:785-792.

14. Wang SY, Chen CT, Sciarappa W, Wang CY, Camp MJ. Fruit quality, antioxidant capacity, and flavonoid content of organically and conventionally grown blueberries. J Agric Food Chem 2008;56:5788-5794.

15. Araya H, C. C, Herrera C. Capacidad antioxidante de frutas y verduras cultivados en Chile. Arch Latinoam Nutr 2006;56:361-365.

16. Speisky H. Frutas y verduras como fuentes de antioxidantes naturales: Su importancia para la salud humana (Fruits and vegetables as sources of natural antioxidants: Its role for human health). In: Chile. Ministerio de Agricultura; Universidad de Chile I, ed. Contribución de la política agraria al consumo de frutas y verduras en Chile: Un compromiso con 
la nutrición y la salud de la población (ed Olivares, S., Leporati, M., Villalobos, P., Barria, L.). Santiago: Maval Ltda.; 2008: pp 45-60.

17. Di Renzo L, Di Pierro D, Bigioni M, Sodi V, Galvano F, Cianci R, La Fauci L, De Lorenzo A. Is antioxidant plasma status in humans a consequence of the antioxidant food content influence? Eur Rev Med Pharmacol Sci. 2007;11:185-192.

18. Hui-Yu H, Chen-Kang C, Tim T, Ju-Jen H, Wei-Wei $\mathrm{C}$, Ying-Chieh T. Antioxidant activities of various fruits and vegetables produced in Taiwan. Internat J Food Sciences Nutr 2004;55:423-429.

19. Gomez-Romero M, Arraez-Roman D, SeguraCarretero A, Fernandez-Gutierrez A. Analytical determination of antioxidants in tomato: typical components of the Mediterranean diet. J Sep Sci 2007;30:452-461.

20. Periago MJ, Garcia-Alonso J, Jacob K, Olivares AB, Bernal MJ, Iniesta MD, Martinez C, Ros G. Bioactive compounds, folates and antioxidant properties of tomatoes (Lycopersicum esculentum) during vine ripening. Int J Food Sci Nutr 2008:1-15.
21. Bruckdorfer KR. Antioxidants and CVD. Proc Nutr Soc 2008;67:214-222.

22. Ortega RM. Importance of functional foods in the Mediterranean diet. Public Health Nutr 2006;9:1136-1140.

23. Torres C, Guzman L, Moore-Carrasco R, Palomo I. Efecto antitrombótico, una característica poco conocida de las frutas y hortalizas Revista Chil Nutr 2008;35:10-17.

24. Rojas A, Villalobos P, Carrasco G. La dieta mediterránea base fundamental de la proyección de chile como "potencia agroalimentaria de nivel mundial". En: Dieta Mediterránea: prevención de las Enfermedades Cardiovasculares Palomo I, Leiva E, Vásquez M (Eds). Editorial Universidad de Talca 2007 pp 129-149.

25. Una política de Estado para la Agricultura Chilena, Periodo 2000 - 2010. Disponible en www.minagri. gob.cl/politica/politic2000-2010.pdf (Consultado el 09/10/2008).

26. Programa 5 al Día en Chile. Disponible en www.inta. cl/5aldia/5aldia.pdf. (Consultado el 11/10/2008). 